\title{
INFLUÊNCIA DA RAZÃO DE RECICLO NO DESEMPENHO DE UM SISTEMA MBBR DE PRÉ-DESNITRIFICAÇÃO
}

\author{
S.M.S. CAO ${ }^{1}$, J.P. BASSIN e M. DEZOTTI \\ ${ }^{1}$ Universidade Federal do Rio de Janeiro, COPPE, Programa de Engenharia Química \\ E-mail para contato: scao@peq.coppe.ufrj.br
}

\begin{abstract}
RESUMO - Um efluente industrial com alta salinidade foi tratado em um sistema MBBR de pré-desnitrificação durante 400 dias. O sistema foi avaliado em termos de remoção de matéria orgânica, nitrogênio e toxicidade. Embora as características do efluente industrial tenham apresentado grande variação ao longo do tempo, a remoção de DQO, COD e nitrogênio amoniacal atingiram níveis acima de $95 \%$ durante a operação estável do sistema. O aumento da razão de reciclo de 200 a $400 \%$ foi acompanhado pelo aumento da eficiência de remoção de nitrogênio, que atingiu valores máximos próximos aos teoricamente possíveis de serem obtidos nas respectivas razões de reciclo. Com o aumento da razão de reciclo, observou-se um aumento da proporção de DQO removida na zona anóxica em relação ao total removido, implicando no aumento da taxa de nitrificação no reator aeróbio. A toxicidade do efluente foi reduzida após o processo biológico. A aplicação de microfiltração seguida de osmose inversa permitiu tornar o efluente tratado apto ao reúso.
\end{abstract}

\section{INTRODUÇÃO}

Os efluentes industriais apresentam considerável quantidade de matéria orgânica e nutrientes em sua composição. O impacto adverso da matéria orgânica é sabido de longa data. Por sua vez, altas concentrações de nitrogênio podem causar a eutrofização dos corpos d'água receptores, evidenciada pelo crescimento acelerado de algas e outras plantas aquáticas. Como consequência, esse fenômeno provoca a mortandade dos seres aquáticos além de ocasionar a poluição do recurso hídrico. Assim, é imprescindível um tratamento eficiente para evitar riscos para a biota.

O tratamento biológico constitui a base dos tratamentos de águas residuárias de todo mundo, devido a sua boa capacidade de remoção dos constituintes tóxicos e as suas vantagens econômicas quando comparado a outras formas de tratamento de efluentes. Apesar da sua larga utilização, os processos biológicos são constantemente investigados na literatura com objetivo aumentar as eficiências de remoção.

Os processos biológicos com biomassa aderida, também conhecidos como processos com biofilme, têm como principal vantagem em detrimento aos com biomassa em suspensão a utilização de uma área menor para sua implementação. Além disso, a quantidade de biomassa ativa pode ser maior nesses reatores, permitindo elevadas taxas de remoção e reduzido tempo de retenção hidráulico (TRH). 


\section{9 a 22 de outubro de 2014 \\ Florianópolis/SC}

Existem vários tipos de reatores com biofilme, dentre os quais o reator de leito móvel com biofilme (MBBR) tem ganhado destaque por apresentar boas remoções de DQO e nitrogênio (nitrificação/desnitrificação).A principal característica deste sistema é que a biomassa cresce aderida a um meio de suporte móvel, que lhe confere a vantagem de não haver entupimento do leito, além de apresentar menor perda de carga em relação aos reatores de leito fixo (RUSTEN et al. 2006).

Os meios de suporte apresentam alta mobilidade em todo volume útil de reator. Como a biomassa fica contida nos meios de suporte, o tanque de sedimentação de lodo pode ser mais compacto (ØDEGAARD, 2006). Além disso, o MBBR pode ser aplicado a estações de tratamento já existentes, sendo necessárias pequenas adaptações (SALVETTI et al., 2006).

A remoção biológica de nitrogênio é alcançada por meio de duas zonas distintas: uma zona aeróbia que é responsável pela nitrificação (oxidação do nitrogênio amoniacal em nitrato), e uma zona anóxica, responsável pela desnitrificação, reduz o nitrato (oxidado na zona aeróbia) a nitrogênio elementar (METCALF \& EDDY, 2003).

Além do tratamento adequado de seus efluentes, as indústrias buscam tecnologias capazes de promover a reutilização das águas nos processos produtivos, visando um consumo sustentável. Neste contexto, os processos de separação por membranas (PSM) vêm se destacando. As membranas comumente utilizadas para tratamento de águas residuárias são as de microfiltração, ultrafiltração, nanofiltração e osmose inversa. Na prática do reúso, a osmose inversa se destaca entre os outros PSM (MADAENI et al., 2010).O uso dos processos de separação por membranas para reúso industrial esta sendo muito relatado na literatura. Apesar disso, ainda são necessários estudos mais aprofundado sobre o tema, que é muito importante na atualidade.

Neste contexto, o presente estudo teve como objetivo avaliar a remoção de matéria orgânica e nitrogênio de um efluente industrial utilizando a tecnologia do MBBR, numa configuração de prédesnitrificação. $\mathrm{O}$ efeito da recirculação de nitrato para a zona anóxica também foi investigado. Além disso, foi avaliada a possibilidade de reúso por meio dos processos de separação por membranas de microfiltração e osmose inversa.

\section{MATERIAIS E MÉTODOS}

O efluente industrial utilizado neste estudo foi fornecido pela indústria química Bayer S/A, localizada em Belford Roxo - RJ e foi coletado do decantador primário de sua estação de tratamento, sem nenhum tipo de tratamento secundário. Esta empresa produz inseticidas, fungicidas, acaricidas e herbicidas, além de matérias-primas básicas para o poliuretano, como Anilina, Poliuretanos Multipropósitos (PU-M) e Metileno difenildiisocianato (MDI). Devido à produção destes produtos químicos, o efluente industrial apresenta alta complexidade, evidenciado pela sua recalcitrância e alta concentração salina. Além disso, o efluente industrial apresentou ao longo do estudo grande variabilidade dos principais parâmetros analisados devido às modificações sofridas pelo próprio processo industrial. 


\subsection{Unidade Experimental}

Dois reatores de leito móvel com biofilme (MBBR), dispostos em série na configuração de prédesnitrificação, foram operados de modo contínuo. O reator anóxico $\left(\mathrm{MBBR}_{1}\right)$, que continha volume reacional de 1 litro, foi agitado com auxilio do borbulhamento de um gás inerte $\left(\mathrm{N}_{2}\right)$ controlado por meio de um rotâmetro. A vazão de gás foi mantida em 1,5 L/min, valor que permitiu completa homogeneização do biorreator e garantiu concentração nula de oxigênio na zona anóxica. No reator aeróbio $\left(\mathrm{MBBR}_{2}\right)$ o volume reacional foi de $2 \mathrm{~L}$. A vazão de ar comprimido foi controlada por um rotâmetro e mantida aproximadamente em $4 \mathrm{~L} / \mathrm{min}$. $\mathrm{O} \mathrm{pH}$ foi ajustado na faixa entre $\mathrm{XX}-\mathrm{XX}$ em ambos os reatores. A unidade experimental está exemplificada na Figura 1.

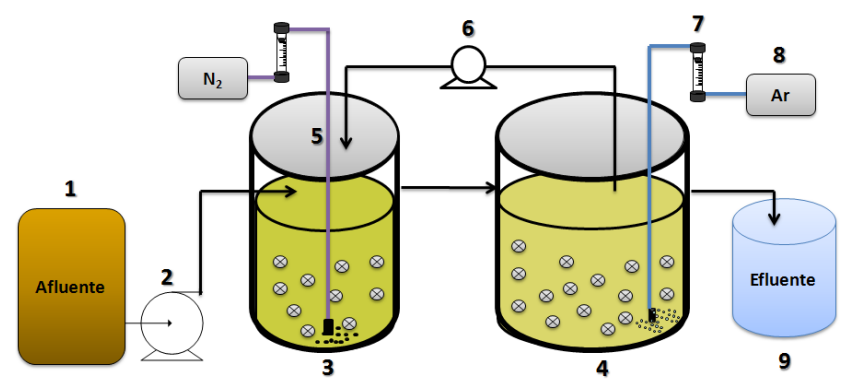

Figura 1 - Representação esquemática do sistema de pré-desnitrificação: 1-Afluente, 2-Bomba peristáltica, 3-Reator Anóxico - $\mathrm{MBBR}_{1}$, 4-Reator Aeróbio - $\mathrm{MBBR}_{2}$, 5- $\mathrm{N}_{2}$, 6-Reciclo, 7-Rotâmetro, 8-Ar comprimido, 9-Efluente tratado.

O sistema foi inoculado com lodo ativado proveniente da Estação de tratamento de Esgotos Alegria, localizada no bairro do Caju, Rio de Janeiro - RJ. Assim, o sistema foi operado continuamente por aproximadamente 400 dias. Foram testadas 3 razões de recirculação distintas, correspondentes aos regimes 1, 2 e 3, exemplificado na Tabela 1. Além disso, a tabela apresenta as características do regime operacional em função do aporte de carga orgânica volumétrica (COV), além da carga orgânica superficial (COS), calculados com base no volume reacional do $\mathrm{MBBR}_{1}$ e na área superficial para crescimento do biofilme, respectivamente.

Tabela 1: Características dos regimes de operação do sistema de pré-desnitrificação

\begin{tabular}{|c|c|c|c|c|}
\hline Regime & Razão de Reciclo ${ }^{1}$ & Tempo (Dias) & $\mathrm{COV}\left(\mathrm{kg} \mathrm{DQO} / \mathrm{m}^{3}\right.$.dia) & COS (g DQO/m².dia) \\
\hline 1 & $200 \%$ & 176 & $0,95 \pm 0,2$ & $3,89 \pm 0,9$ \\
\hline 2 & $300 \%$ & 91 & $0,95 \pm 0,3$ & $3,20 \pm 0,9$ \\
\hline 3 & $400 \%$ & 143 & $0,72 \pm 0,1$ & $2,51 \pm 0,5$ \\
\hline
\end{tabular}

Os reatores operaram com tempo de retenção hidráulico (TRH) de 12 horas para o $\mathrm{MBBR}_{1}$ e 24 horas para o $\mathrm{MBBR}_{2}$. Em ambos reatores foi utilizado o suporte k1, fornecido pela AnoxKaldnes®, com fração de enchimento de $50 \%$ em relação ao volume total do reator, de modo que área disponível para o crescimento microbiano correspondeu a $250 \mathrm{~m}^{2} / \mathrm{m}^{3}$.

\subsection{Quantificação analítica}




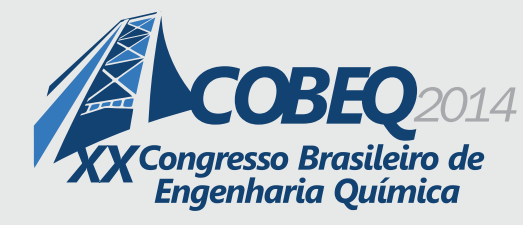

\section{9 a 22 de outubro de 2014 \\ Florianópolis/SC}

A análise dos parâmetros de entrada e saída dos reatores foi realizada para acompanhar a operação do sistema. Os parâmetros analisados foram demanda química de oxigênio (DQO), carbono orgânico dissolvido (COD), nitrogênio total, amônio e cloreto, os quais foram determinados de acordo com Standard Methods (1995). O teste de toxicidade foi realizado de acordo com a metodologia normalizada pela Associação Brasileira de Normas Técnicas (ABNT NBR 15411-2:2006)..

\section{RESULTADOS}

\subsection{Remoção de Matéria Orgânica}

O sistema de pré-desnitrificação foi alimentado continuamente por aproximadamente 400 dias. A formação do biofilme foi observada nos 30 primeiros dias de operação do sistema. Nesse período, a eficiência de remoção de matéria orgânica, analisada em termos de DQO e COD (Figura 2a, 2b), foi aumentada gradativamente, até atingir eficiências de remoção superior a 80\%. Após esse período, apesar da grande variabilidade da DQO e do COD afluentes, a saída permaneceu quase que invariável, em baixos valores, indicando que o biofilme formado estava colonizado por microrganismos bem adaptados as variações de carga orgânica oriundas da composição variável do efluente. A relação DQO/COD do afluente variou numa faixa entre 2 e 7 , compatível com os valores encontrados na literatura para outros efluentes industriais (SANT'ANNA JR., 2013).

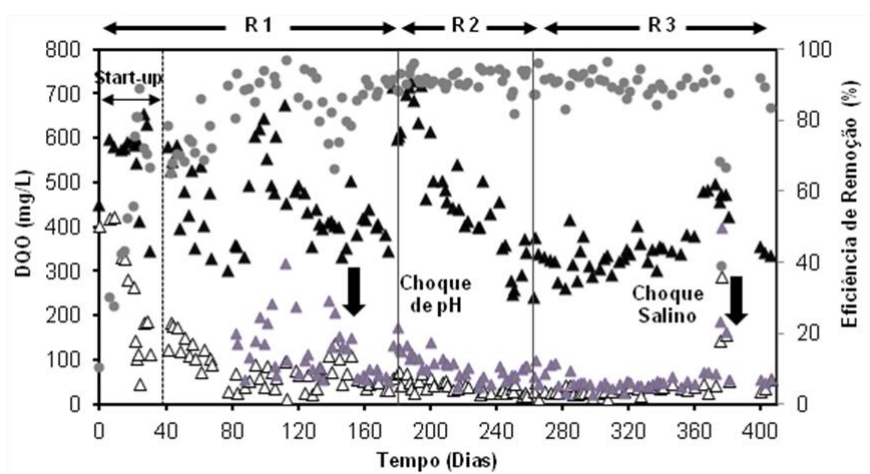

(a)

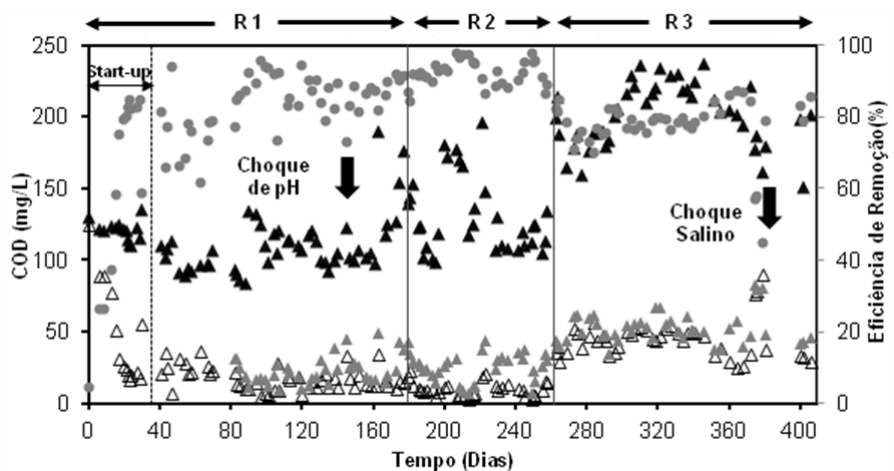

(b)

Figura 2 (a) e (b) - Afluente ( $\mathbf{\Delta})$, Efluente $\operatorname{MBBR}_{1}(\Delta)$, Efluente $\operatorname{MBBR}_{2}(\Delta)$ e Eficiência de remoção $\mathrm{DQO}$ e $\operatorname{COD}(\bullet)$, respectivamente.

Como se pode observar nas Figuras $2 \mathrm{a}$ e $2 \mathrm{~b}$, ocorreu um choque acidental de $\mathrm{pH}$ no dia 140 de operação. Esse parâmetro foi reduzido a 2, muito abaixo dos valores recomendados pela literatura para tratamento biológico que encontram-se entre 6,5 e 8 (METCALF \& EDDY, 2003). Houve uma queda de eficiência de DQO e COD, que foi reduzida de valores superiores a $85 \%$ para $66 \%$ e $75 \%$, respectivamente. Outra perturbação no sistema (choque de salinidade) afetou de forma significativa a remoção de matéria orgânica. A concentração salina foi aumentada de valores em torno de 3,7 a 6,6 $\mathrm{gCl}^{-} / \mathrm{L}$ para salinidade superior a $45 \mathrm{gCl}^{-} / \mathrm{L}$ no dia 372 de operação, mantido nesta condição durante 3 dias. Neste período também houve uma significava queda de eficiência de remoção de DQO e COD, sendo mais pronunciada que a causada pelo choque de $\mathrm{pH}$. 


\section{9 a 22 de outubro de 2014 \\ Florianópolis/SC}

É importante salientar que apesar do efluente industrial apresentar salinidade relativamente alta (3,7 a 6,6 $\left.\mathrm{gCl}^{-} / \mathrm{L}\right)$, esta não apresentou inibição sobre o processo biológico. Em ambientes de alta salinidade pode ocorrer remoção de matéria orgânica sem dificuldades devido à capacidade de adaptação de certos micro-organismos as essas condições. ARTIGA et al. (2008) observou altas remoções de matéria orgânica após a aclimatação do lodo a alta salinidade.

Após o período de aclimatação, observou-se que maior parte da DQO e COD foi removida no reator primeiro reator $\left(\mathrm{MBBR}_{1}\right)$. As Figuras $3 \mathrm{a}$ e $3 \mathrm{~b}$ mostram a contribuição do reator $\mathrm{MBBR}_{1} \mathrm{e}$ reator $\mathrm{MBBR}_{2}$ na remoção de $\mathrm{DQO}$ e $\mathrm{COD}$, respectivamente. $\mathrm{O}$ consumo de matéria orgânica prioritariamente no primeiro reator foi devido à ação das bactérias heterotróficas, responsáveis pela desnitrificação, que utilizam a matéria orgânica como doador de elétrons para redução do nitrato a nitrogênio gasoso. Essa maior remoção no $\mathrm{MBBR}_{1}$ possibilitou um meio com baixa concentração de carbono orgânico, propício para realização da nitrificação no $\mathrm{MBBR}_{2}$. Nessas condições, observou-se um aumento da taxa de nitrificação.

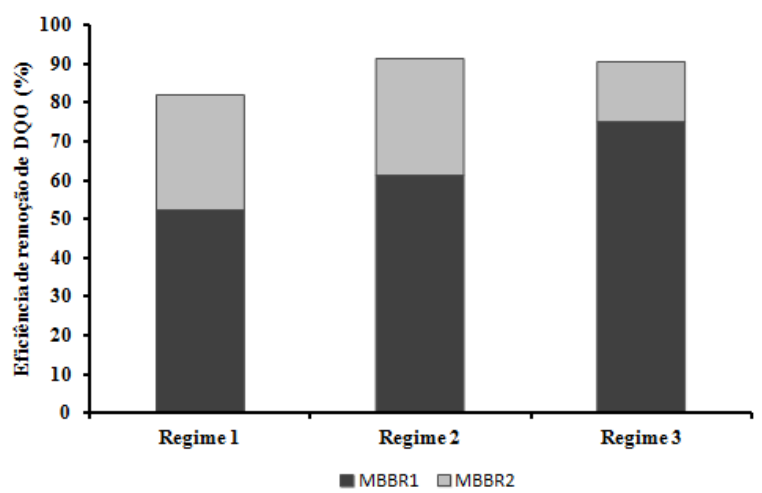

(a)

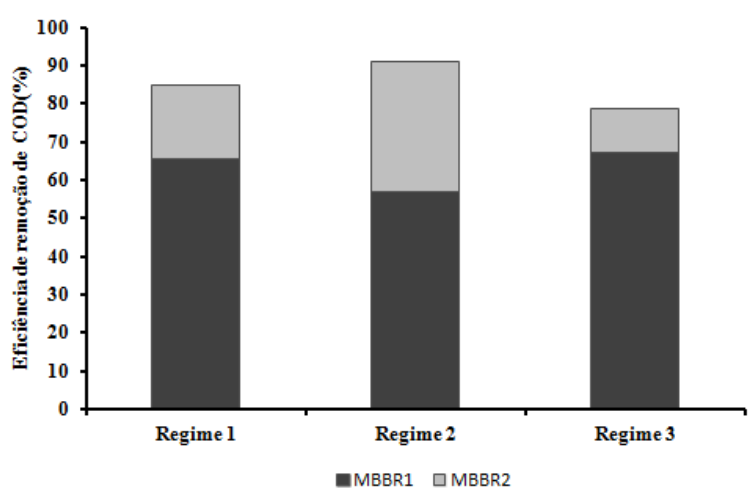

(b)

Figura 3 (a) e (b) - Contribuição do $\mathrm{MBBR}_{1}$ e $\mathrm{MBBR}_{2}$ para a remoção matéria orgânica, evidenciado por meio do balanço de massa para a DQO e COD, respectivamente.

\subsection{Remoção de Nitrogênio (N)}

As Figuras 4 (a) e 4 (b) apresentam os gráficos referentes à remoção de nitrogênio amoniacal (nitrificação) que ocorre no segundo reator $\left(\mathrm{MBBR}_{2}\right)$ e a remoção de nitrogênio (desnitrificação) que ocorre no primeiro reator $\left(\mathrm{MBBR}_{1}\right)$. Como se pode observar, há um perfil de aumento da eficiência de remoção com o tempo. As remoções de nitrogênio amoniacal $\left(\mathrm{NH}_{4}{ }^{+}\right)$em todos os regimes operacionais foram superiores a 90\%, mantendo-se quase que invariável. No entanto, a eficiência de remoção de nitrogênio foi proporcional à quantidade de nitrato recirculada para o primeiro reator $\left(\mathrm{MBBR}_{1}\right)$. Assim, houve um aumento gradual da eficiência de remoção de $\mathrm{N}$, devido aumento gradual da razão de reciclo, de $200 \%$ a 400\%. No regime 1, a remoção máxima esperada de nitrogênio era de $66,7 \%$, sendo que o sistema atingiu remoções médias de 57,6\%. No segundo regime, o máximo que o sistema poderia remover era de $75 \%$ em virtude da razão de reciclo ter sido de $300 \%$. O sistema removeu $71,3 \%$ de nitrogênio. A mesma tendência foi observada no terceiro regime, no qual o máximo esperado era de $80 \%$ de remoção de $\mathrm{N}$ e atingiu-se em média $78,1 \%$ de remoção de nitrogênio. 


\section{9 a 22 de outubro de 2014 \\ Florianópolis/SC}

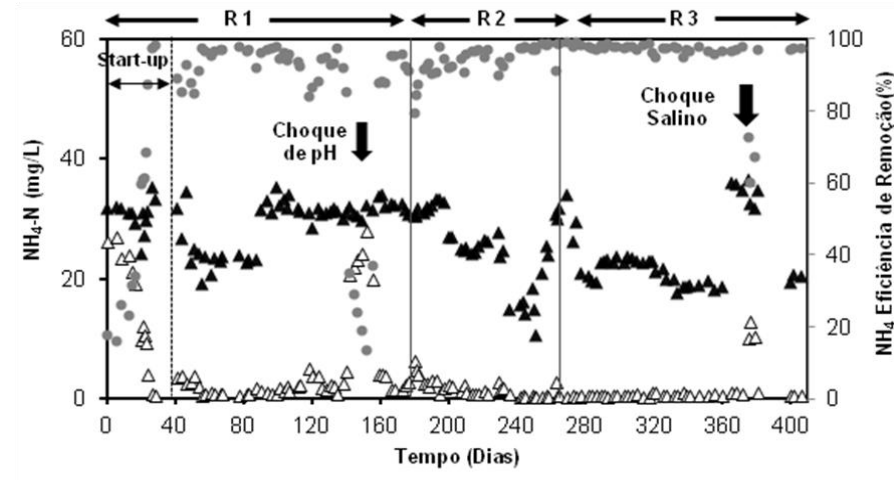

(a)

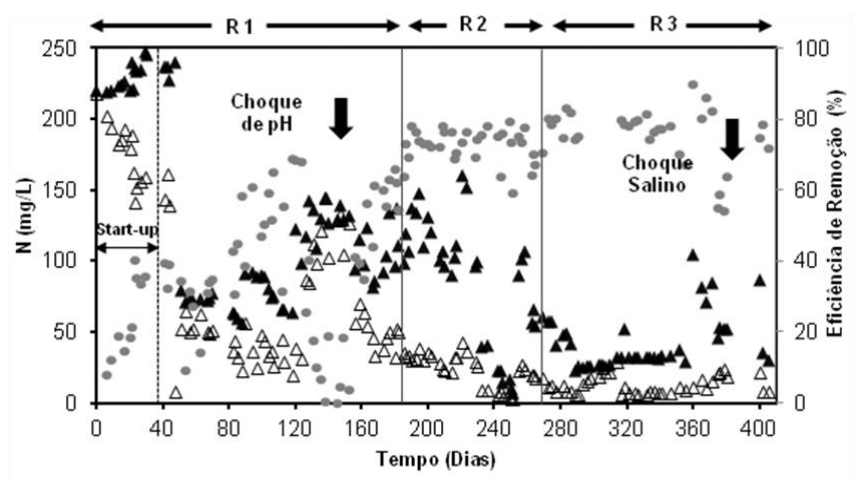

(b)

Figura 4 - (a) Afluente ( $\Delta$ ), Efluente $\mathrm{MBBR}_{2}(\Delta)$ e Eficiência de remoção $\mathrm{NH}_{4}^{+}(\bullet)$, (b) Afluente (entrada do $\left.\operatorname{MBBR}_{1}\right)(\boldsymbol{\Lambda})$, Efluente (saída do $\left.\mathrm{MBBR}_{2}\right)(\Delta)$ e Eficiência de remoção de $\mathrm{N}(\bullet)$.

O choque de $\mathrm{pH}$ afetou bruscamente a eficiência remoção de nitrogênio amoniacal, a qual caiu para valores inferiores a $13 \%$. Do mesmo modo, nesse período não foi verificada desnitrificação. Quanto ao choque de salinidade, conforme demonstrado nas Figuras 4(a) e 4(b), o mesmo afetou com menor intensidade a eficiência de nitrificação e remoção de nitrogênio do sistema.

Um fator importante na remoção de nitrogênio é a relação DQO/Nitrogênio (DQO/N). No reator $\mathrm{MBBR}_{2}$, responsável pela nitrificação, é desejável uma baixa relação DQO/N. Em contrapartida, no reator responsável pela desnitrificação, uma elevada relação DQO/N é recomendada na literatura. A razão DQO/N afluente variou de acordo com o regime estudado, situando-se na faixa entre 4,3-9,5 $\mathrm{mg}-\mathrm{DQO} / \mathrm{mg}-\mathrm{N}$. Este valor está dentro do mínimo estabelecido na literatura para desnitrificação (ZHEN et al., 2007; GE et al., 2012).

\subsection{Biomassa aderida ao suporte}

A biomassa aderida ao suporte foi quantificada por meio da análise de sólidos suspensos voláteis (SSV) ao longo dos regimes estudados. A concentração de biomassa no reator $\mathrm{MBBR}_{1}$ variou em média de 7,6-9,1 gSSV/L e no segundo reator $\mathrm{MBBR}_{2}$ variou de 7,6-11,7 gSSV/L. A concentração de SSV encontrada nesta pesquisa esta próxima as relatadas na literatura, na qual estabelece valores típicos de SSV em sistema MBBR de 2-8 gSSV/L (RUSTEN et al. 1995). Além disso, as análises mostram que a biomassa aderida é majoritariamente constituída por material orgânico (sólidos biológicos), evidenciada pela relação SSV/SST de 0,88 para $\mathrm{MBBR}_{1}$ e 0,9 para o $\mathrm{MBBR}_{2}$.

\subsection{Toxicidade}

Foram realizados testes de toxicidade para o afluente industrial e as saídas do sistema biológico por meio da bactéria Vibrio Fischeri. Este teste consiste na exposição do micro-organismo teste ao agente toxico. A toxicidade foi expressa em termos de CE50, que é a concentração efetiva do agente tóxico que causa $50 \%$ de redução na quantidade de luz emitida pelo microrganismo teste. A Tabela 2 apresenta os valores médios de CE50 encontrados nos três regimes de operação. Como se pode 
observar, o efluente do primeiro reator já não apresentou toxicidade aguda, indicando que o tratamento biológico reduziu a toxicidade do efluente industrial.

Tabela 2: Valor da CE50 em ensaios de toxicidade aguda com Vibrio fischeri

\begin{tabular}{cccc}
\hline \multirow{2}{*}{ Regimes de operação } & Afluente & $\begin{array}{c}\mathrm{CE}_{50}(\%) \\
\text { Saída MMBR }\end{array}$ & Saída MBBR $_{2}$ \\
\hline 1 & 63 & STA & STA \\
2 & 68 & STA & STA \\
3 & 71 & STA & STA \\
\hline
\end{tabular}

STA: Sem toxicidade aguda

\subsection{Processos de separação por membranas}

Por meio das análises físico-químicas da saída do sistema biológico, verificou-se a necessidade do tratamento terciário, a fim de se viabilizar o reúso do efluente na indústria. Desta forma, o efluente foi avaliado após a microfiltração e osmose inversa. A microfiltração foi essencial para viabilizar o uso da osmose inversa, sem que esta sofresse potencialmente com a incrustação. $O$ índice de densidade de sedimentos (SDI) foi determinado com o objetivo de se avaliar o potencial de incrustação da membrana de osmose inversa. O efluente da saída do sistema biológico apresentou SDI de 6,7, valor que, segundo BAKER (2004), denota aplicação inviável para aplicação direta no módulo de osmose inversa. Por sua vez, o efluente microfiltrado apresentou SDI médio de 2,1, o qual indica que o sistema de osmose inversa pode funcionar por vários meses sem a necessidade de limpezas constantes.

A Tabela 3 sumariza os valores obtidos para alguns parâmetros em diversos estágios do tratamento (entrada e saída do processo biológico, microfiltração e osmose inversa). Após a combinação destes processos, foi possível obter um efluente apto para o reúso industrial, o qual tem potencial para, por exemplo, ser aplicado em torres de resfriamento ou caldeiras.

Tabela 3: Resultados obtidos durante os diferentes tratamentos adotados (biológico, MF e OI)

\begin{tabular}{lcccccc}
\hline \multirow{2}{*}{ Parâmetros } & \multicolumn{2}{c}{ MBBR } & \multicolumn{3}{c}{ Microfiltração } & \multicolumn{2}{c}{ Osmose Inversa } \\
& Entrada & Saída & Entrada & Saída & Alimentação & Permeado \\
\hline COD (mg/L) & 207 & 45 & - & - & 50,7 & 1,63 \\
DQO (mg/L) & 357 & 33 & 33 & 32 & - & - \\
Amônio (mg/L) & 26 & 0,6 & - & - & - & - \\
Nitrogênio (mg/L) & 43 & 13 & - & - & 15,2 & 3,07 \\
Condutividade & 18711 & 18711 & 18711 & 18212 & 18153 & 0,15 \\
(mS/cm) & 0,221 & 0,212 & 0,269 & 0,182 & - & - \\
SST (g/L) & 0,13 & 0,13 & 0,13 & 0,14 & - & - \\
SSV (g/L) & & & & & & \\
\hline
\end{tabular}

\section{CONCLUSÕES}

O estudo voltado ao tratamento do efluente de indústria química evidenciou que o sistema de pré-desnitrificação foi eficiente na remoção de matéria orgânica e nitrogênio. A eficiência de remoção de nitrogênio esteve diretamente relacionada à razão de reciclo de nitrato a partir do reator aeróbio 


\section{9 a 22 de outubro de 2014 \\ Florianópolis/SC}

para o anóxico. O teste de toxicidade apontou que ao final do tratamento biológico o efluente não apresentou toxicidade aguda. Além disso, após a microfiltração, o efluente se mostrou apto para processo de osmose inversa, com SDI médio de 2. A combinação do tratamento biológico com os processos de separação por membranas proporcionou um efluente adequado ao reúso industrial, podendo ser reutilizado, por exemplo, em caldeiras ou em torres de resfriamento.

\section{REFERENCIAS}

ABNT NBR 15411-2, 2006, Ecotoxicologia Aquática - Determinação do efeito inibitório de amostras de água sobre a emissão de luz de Vibrio Fischeri (ensaio de bactéria luminescente) parte 2: Método utilizando bactérias desidratadas.

APHA, 1995, Standard Methods for the Examinacion of Water and Wastewater.

ARTIGA, P., GARCIA-TORIELLO, G., MENDEZ, R., GARRIDO, J.M., 2008, "Use of a hybrid membrane bioreactor for the treatment of saline wastewater from a fish canning factory", Desalination, V.221, 518-525.

BAKER, R. W., 2004, Membrane technology and applications, 2nd ed., John Wiley \& Sons Ltd, pp. 217-219, California, EUA.

GE, S., PENG, Y., WANG, S., LU, C., CAO, X., ZHU, Y., 2012, "Nitrite accumulation under constant temperature in anoxic denitrification process: The effects of carbon sources and COD/NO 3 -N", Bioresource Technology, V.114, pp. 137-143.

LAZAROVA,V., MANEM J., 1995, "Biofilm characterization and activity analysis in water and wastewater treatment", Water Research, V.29, p.2227-2245.

MADAENI, S. S., SAMIEIRAD, S., 2010, “Chemical Cleaning of Reverse Osmosis Membrane Fouled by Wastewater", Desalination, V.257, 80-86.

METCALF \& EDDY, 2003, Wastewater engineering: treatment, disposal and reuse, $4^{\mathrm{a}} \mathrm{ed}$., New York: McGraw Hill.

ØDEGAARD, H., 2006, "Innovations in wastewater treatment: the moving bed biofilm process", Water Science Technology, V.53, pp. 17-33.

RUSTEN, B., EIKEBROKK, B., ULGENES, Y., LYGREN, E., 2006, "Design and operations of the Kaldnes moving bed biofilm reactors", Aquacultural Engineering, V.34, pp. 322-331.

RUSTEN, B., HEM, L., ØDEGAARD, H., 1995, "Nitrification of municipal wastewater in moving-bed biofilm reactors", Water \& Environment Research, V. 67, pp. 75-86.

SALVETTI, R., AZZELLINO, A., CANZIANI, R., BONOMO, L., 2006, "Effects of temperature on tertiary nitrification in moving-bed biofilm reactors", Water Research, V. 40, pp. 29812993.

SANT'ANNA JR., 2013, Tratamento Biológico de Efluentes, $2^{\text {a }}$ Ed., Editora: Interciência, Rio de Janeiro, Número de páginas: 424.

ZHEN, P.Y., YONG, M., YING, W.S., 2007, "Denitrification potential enhancement by addition of external carbon sources in a pre-denitrification process", Journal Environmental Sciences, V.19, pp. 284-289. 